\title{
Time-Coordination Strategies and Control Laws for Multi-Agent Unmanned Systems
}

\author{
Javier Puig-Navarro*, Naira Hovakimyan ${ }^{\dagger}$ \\ University of Illinois at Urbana-Chamapaign, Urbana, IL, 61801, USA \\ B. Danette Allen ${ }^{\ddagger}$ \\ NASA Langley Research Center, Hampton, VA, 23681, USA
}

\begin{abstract}
Time-critical coordination tools for unmanned systems can be employed to enforce the type of temporal constraints required in terminal control areas, ensure minimum distance requirements among vehicles are satisfied, and successfully perform coordinated missions. In comparison with previous literature, this paper presents an ampler spectrum of coordination and temporal specifications for unmanned systems, and proposes a general control law that can enforce this range of constraints. The constraint classification presented considers the nature of the desired arrival window and the permissible coordination errors to define six different types of time-coordination strategies. The resulting decentralized coordination control law allows the vehicles to negotiate their speeds along their paths in response to information exchanged over the communication network. This control law organizes the different members in the fleet hierarchically per their behavior and informational needs as reference agent, leaders, and followers. Examples and simulation results for all the coordination strategies presented demonstrate the applicability and efficacy of the coordination control law for multiple unmanned systems.
\end{abstract}

\section{Introduction}

Recent developments in unmanned systems (UxSs) technologies have opened vast opportunities in scientific research, $\frac{1}{3} \sqrt[3]{3}$ remote monitoring and control, $\frac{14}{4}$ rapid assessment of catastrophic events, $\frac{5}{5}$ search and rescue operations, $\underset{6}{[7]}$ cartography, $\stackrel{[8]}{a}$ and cinematography, to name a few examples. Not only have UxSs eliminated the constraints and limitations of manned vehicles, but also their relatively low cost, miniaturization levels, and ease of deployment have popularized their use in the aforementioned areas. Moreover, fleets of cooperating UxSs can be used to exploit synergistic behaviors among vehicles, remove the risk of single-point failures, improve flexibility, increase redundancy and reliability, achieve blanket coverage of larger areas, and often reduce mission execution times.

A fundamental component to achieve these goals is the availability of cooperative control strategies that are robust to temporary communication dropouts, packet loss, and external disturbances. Additionally, these cooperative strategies shall ensure safe inter-vehicle separation, while being able to adapt to changing mission goals, fleet size, and vehicle diversity. Technical advances in time coordination have the potential to realize new transportation paradigms that hold promise for revolutionary changes in other fields such as air traffic management $\frac{10}{10}$ mail services, and ground transportation systems.

Inspired by these challenges, previous work ${ }^{11}[12$ developed a set of algorithms for time-critical cooperative path-following control of a fleet of Unmanned Aerial Vehicles (UAVs) that exchange information over a communication network. The resulting framework divides the fleet into leaders and followers and guarantees that the UAVs follow their assigned trajectories, while meeting temporal and spatial constraints. Moreover,

\footnotetext{
*Graduate Student, Dept. of Aerospace Engineering, 104 S. Wright St., Urbana, IL, AIAA Student Member.

${ }^{\dagger}$ Professor, Dept. of Mechanical Science and Engineering, 1206 W. Green St., Urbana, IL, AIAA Fellow.

${ }_{\ddagger}^{\ddagger}$ Senior Technologist for Intelligent Flight Systems, Crew Systems \& Aviation Operations Branch, MS 492, AIAA Senior Member.
} 
this set of algorithms allows UAVs to compensate for off-nominal situations by negotiating the speed along their paths.

In this paper, we focus on the time-coordination aspect of this framework. First developments 13 considered tight coordination constraints among vehicles, leaving no room for bounded coordination coordination errors among UxSs. Later work ${ }^{14}$ combined tight coordination constraints with a variety of temporal constraints, such as a specific time of arrival or a desired window of arrival for one or more points along the assigned paths. To achieve this, the reference agent was introduced 14 as a means to impose temporal specifications on the fleet of UxSs. This paper maintains the reference agent and expands the previous spectrum of time-coordination strategies.14 In particular, loose coordination constraints are introduced and combined with the previous set of coordination and temporal assignments, resulting in six general timecoordination strategies. The importance of loose coordination constraints relies in the fact that it explicitly allows bounded errors in the coordination among vehicles. The practicality of each strategy is illustrated through a real-world scenario. This paper proposes a new and broader control law that relies on the decision logic of a set of link weights to impose the desired set of coordination and temporal constraints. Moreover, the algorithm presented here provides all agents in the fleet with disturbance rejection capabilities. As a result, contrary to previous publications, leaders do not necessarily need to be virtual agents. The paper also presents simulation results that highlight the importance of imposing the appropriate set of temporal and coordination constraints. In fact, the paper provides numerical evidence that loose coordination is preferable (if this type of constraint is admissible for the mission in question) over tight coordination since it leads to smaller speed corrections, and thus a reduction in fuel consumption. Numerical evidence supporting a similar prioritization for the types of temporal constraints is also provided.

This paper is organized as follows. Section II presents a brief overview of the background and algorithms required to perform multi-vehicle cooperative missions, and their relation with the time coordination strategies and protocols presented in this paper. Section III characterizes the time coordination problem as a combination of consensus and collective tracking problem, and provides a physical interpretation of the resulting problem formulation. Section IV defines the set of coordination constraints and temporal specifications that lead to the definition of the time coordination strategies presented in this paper. Examples of realistic missions that could be executed with these strategies are provided to motivate the applicability of this work. Section $\nabla$ proposes a novel distributed control law that enforces desired coordination constraints and temporal specifications on the fleet of UxSs. SectionVIincludes numerical simulation results that show the capabilities of the aforementioned control law. Finally, SectionVII concludes the paper with a summary of the findings of this research and recommendations regarding the usage of the time coordination algorithm developed.

\section{Background: Time-Critical Cooperative Path Following}

The goal of the aforementioned time-critical cooperative path-following framework is to safely guide a fleet of UxSs along their assigned trajectories, while meeting predefined spatial and temporal constraints. The spatial constraints ensure vehicles do not collide with other vehicles in the fleet or with fixed obstacles, while temporal constraints enforce a desired schedule on the UxSs. Research in this area regarding ground, 16 underwater, $17 \sqrt[19]{19}$ and aerial 20,22 vehicles has grown significantly in the last decade. The cooperative some of this work and is based on the idea of decoupling space and time, and is composed of three basic elements:

- Trajectory generation algorithm: 23 is responsible for the computation of a geometrical path and a desired speed profile $v_{d, i}(t)$ for each of the $n$ vehicles in the fleet, $i \in \mathcal{I}:=\{1, \ldots, n\}$. Under nominal conditions, this algorithm ensures that UxSs maintain safe inter-vehicle separation, while complying with a set of vehicle-specific dynamic constraints, such as minimum and maximum speed or acceleration. To ensure that minimum spatial clearance among vehicles is maintained at all times, this algorithm provides two alternative solutions: $i$ ) spatial deconfliction that is, the minimum distance between any two points on the paths is greater or equal than a minimum safety threshold; and $i i$ ) temporal deconfliction, which guarantees that the distance between any two vehicles is greater or equal than the minimum spatial clearance at all times, even though their corresponding paths may not be spatially deconflicted. To ensure that the vehicles maintain the desired relative position, and thus maneuvers are collision free, this type of deconfliction requires the vehicles to coordinate along their paths. The trajectory generation algorithm is vehicle specific, since it relies on optimization methods that take explicitly into account an optimization criterion and a set of simplified dynamic constrains. 
- Path following algorithm! ${ }^{12[22]}$ ensures that UxSs converge to and follow their assigned path, regardless of what the speed profile is, as long as it is physically feasible. Note that this algorithm is also vehicle-specific since path-following strategies vary for fixed-wing aircraft, multirotors, or watercraft, to mention but a few examples.

- Time-coordination algorithm: $\frac{\sqrt{12}[14}{14}$ guarantees that vehicles in the fleet reach agreement on some distributed variables of interest. These parameters are referred to as the coordination states and denoted as $x_{i}(t) \in \mathbb{R}, i \in \mathcal{I}$. The coordination states and assigned trajectories capture the objectives of the cooperative mission, such as desired relative position among vehicles and temporal constraints. As in previous formulations, $\frac{12}{14}$ the coordination states are scalar time-variables defined over the interval $\left[0, t_{d, i}^{f}\right]$, where $t_{d, i}^{f}$ is the desired nominal duration of the mission for the $i$ th vehicle. These time-variables are defined such that:

- if any two vehicles $i$ and $j$ agree on their coordination states, namely $x_{i}(t)=x_{j}(t)$, then these two vehicles have the desired relative position along their paths at time $t$ and, thus, satisfy the relative temporal constraints as specified by the trajectory-generation algorithm;

- if $\dot{x}_{i}(t)=1$, then the commanded speed $v_{c, i}(t)$ for the $i$ th vehicle is the desired speed $v_{d, i}(t)$ at time $t$ as specified by the trajectory generation algorithm. Further details on how the coordination-state rate modifies the commanded speed can be found in previous work, $\frac{12}{2}$

- if $x_{i}(t)=t$, then the $i$ th vehicle is at the position specified by the trajectory-generation algorithm $t$ seconds after the initiation of the mission, which implies that, at time $t$ this vehicle strictly meets its individual temporal assignment.

\section{Problem Formulation}

Consider a network of $n$ integrator-agents

$$
\dot{x}_{i}(t)=u_{i}(t), \quad x_{i}(0)=x_{i_{0}}, \quad i \in \mathcal{I},
$$

with dynamic information flow $\mathcal{G}(t):=(\mathcal{V}, \mathcal{E}(t))$, where $\mathcal{V}$ and $\mathcal{E}(t)$ represent the vertices and edges of the communication graph, respectively. In our case, $\mathcal{V}$ is the group of UxSs that participate in the coordinated mission, while $\mathcal{E}(t)$ represents the communication links that are established among UxSs at each moment in time. In the above formulation, $u_{i}(t) \in \mathbb{R}$ is the control input, and $\mathcal{I}$ is the set of unique identification numbers of the UxSs in the fleet.

To enforce temporal constraints on the group of UxSs, we utilize the reference agent introduced in earlier publications. ${ }^{14}$ This agent does not attempt coordination with any fleet member, but determines the reference rate $\dot{x}_{R}(t)$ or desired rate of the mission. The reference rate is defined such that if the mission evolves as initially programmed during the mission planning phase ${ }^{23}$

$$
\dot{x}_{R}(t)=1, \quad x_{R}(0)=0, \quad \forall t \geq 0,
$$

where $x_{R}(t) \in \mathbb{R}$ is the reference state. Occasionally, the dynamics of the reference rate can be modified on the fly to speed up or slow down the progress of the mission, and adapt to possible unplanned scenarios. To this end, we introduce a finite number of rescheduling events $n_{s}$, the corresponding rescheduling times $t_{s, i}$, and constant parameters $\gamma_{s, i}$, with $i \in\left\{0,1, \ldots, n_{s}\right\}$. Then, the dynamics of the reference rate can be expressed as

$$
\dot{x}_{R}(t)=\gamma_{s, i}, \quad x_{R}(0)=0, \quad t_{s, i} \leq t<t_{s, i+1}, \quad i \in\left\{0, \ldots, n_{s}-1\right\},
$$

where $t_{s, 0}=0, \gamma_{s, 0}=1$, and $\dot{x}_{R}(t)=\gamma_{s, n_{s}}$ for all $t \geq t_{s, n_{s}}$. This is summarized in the following assumption:

Assumption 1 The reference rate $\dot{x}_{R}(t)$ is a step function on $\mathbb{R} ! 25$

At this point, we introduce the vehicle hierarchy and associated restrictions on the flow of information, outlined in Figure 1. Observe that this schematic introduces the reference-leader and inter-vehicle communication networks. Continuous arrows correspond with variables that are shared continuously over the network, whereas dashed arrows are associated with parameters that need only be exchanged for a limited amount of time after a rescheduling event occurs. Figure 1 classifies the fleet members in three different categories based on their role in the coordination problem and communication capabilities. 


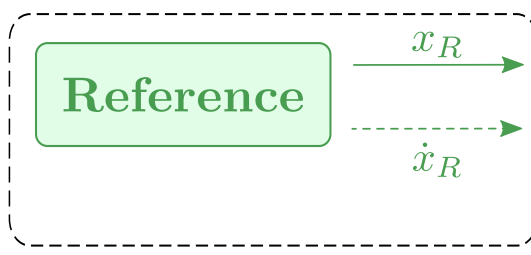

Reference-leader communication network

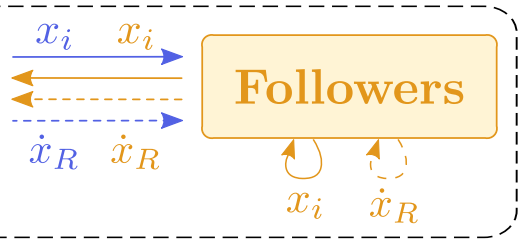

Inter-vehicle

communication network

Figure 1: Communication networks, vehicle hierarchy, and flow of information.

- The reference agent runs as a completely independent member and thus, does not require information from other vehicles. The role of the reference agent is to provide the fleet with the necessary information to enforce temporal constraints. Note that this agent continuously shares the reference state with the leaders, whereas it only sends updates on the reference rate after a rescheduling event occurs. The reference agent can be a physical or a virtual entity, running in a supervisory control facility such as mission control, or onboard the leaders.

- Leaders have direct access to the data from the reference agent, and communicate with other leaders and followers. In fact, leaders exchange their coordination states with other vehicles to achieve coordination. Additionally, each leader can transmit the reference rate to other leaders and followers through a multi-hoop communication protocol. Without loss of generality, vehicles with identification numbers 1 through $n_{\ell}$ are designated as fleet leaders. Hereafter, the set of all leaders will be referred to as $\mathcal{I}_{\ell}:=\left\{1, \ldots, n_{\ell}\right\}$.

- Followers do not have direct communication capabilities with the reference agent. However, each follower shares its coordination state with other followers and leaders. Additionally, upon reception of $\dot{x}_{R}(t)$ they can relay this information to other vehicles through a multi-hoop communication protocol. Vehicles with identification numbers $n_{\ell}+1$ through $n$ are designated as followers. Hereafter, the set of all followers will be referred to as $\mathcal{I}_{f}:=\left\{n_{\ell}+1, \ldots, n\right\}$.

These restrictions on the flow of information aim to capture a rather general scenario where data from the reference agent may not be available to some UxSs. This can occur when some UxSs have limited longrange communications capabilities, or when operations take place in shadowed areas. For instance, low-level flights where line-of-sight communications are unavailable to some of the UxSs. These restrictions are the reason behind the two distinct communication networks illustrated in Figure 1 For the sake of generality, we do not wish to impose any specific structure on the topology of the networks, or assume any a priori knowledge about the amount of data exchanged among group members. Accordingly, the reference-leader communication network satisfies the following general assumptions:

Assumption 2 The reference agent can only exchange information with a time-varying set of leaders, denoted here by $\mathcal{N}_{R}(t) \subseteq \mathcal{I}_{\ell}$.

Assumption 3 Communications are unidirectional and, for simplicity, $x_{R}(t)$ is transmitted continuously and with no delays from the reference agent to the leaders.

Assumption 4 The connectivity of the reference-leaders communication network topology satisfies the persistency of excitation (PE)-like condition ${ }^{26}$

$$
\min _{i \in \mathcal{I}_{\ell}}\left(\frac{1}{T_{R}} \int_{t}^{t+T_{R}} \ell_{R_{i}}(\tau) d \tau\right) \geq \mu_{R} \mathbb{I}_{\mathbf{n}-\mathbf{1}}, \quad \forall t \geq T_{R},
$$

where $\ell_{R_{i}}(t)=1$ if the $i$ th leader receives information from the reference agent at time $t$, and $\ell_{R_{i}}(t)=0$ otherwise. Parameters $T_{R}>0$ and $\mu_{R} \in(0,1]$ characterize the quality of service $(Q o S)$ of the reference-leader communication network.

Assumption 5 The reference agent transmits $\dot{x}_{R}(t)$ continuously, with no delays, and for a limited period of time $T_{R}$ after each rescheduling event. 
Given the QoS of the reference-leader communication network specified in Assumption 4, Assumption 5 guarantees that all leaders receive the updated value of the reference rate after a rescheduling event. Similarly, the inter-vehicle communication network satisfies the following assumptions:

Assumption 6 The ith vehicle can only exchange information with a time-varying set of vehicles, denoted here by $\mathcal{N}_{i}(t) \subset \mathcal{I}, i \in \mathcal{I}$.

Assumption 7 Communications among vehicles are bidirectional and, for simplicity, $x_{i}(t)$ is transmitted continuously and with no delays, $i \in \mathcal{I}$.

Assumption 8 The connectivity of the graph $\mathcal{G}(t)$ that models the inter-vehicle communication network topology satisfies the PE-like condition 26

$$
\frac{1}{n} \frac{1}{T} \int_{t}^{t+T} \mathbf{Q L}(\tau) \mathbf{Q}^{\top} d \tau \geq \mu \mathbb{I}_{\mathbf{n}-\mathbf{1}}, \quad \forall t \geq T,
$$

where $\mathbf{L}(t) \in \mathbb{R}^{n \times n}$ is the piecewise-constant Laplacian of the graph $\mathcal{G}(t)$, and $\mathbf{Q} \in \mathbb{R}^{(n-1) \times n}$ is a matrix satisfying $\mathbf{Q} \mathbf{1}_{\mathbf{n}}=\mathbf{0}$, and $\mathbf{Q} \mathbf{Q}^{\top}=\mathbb{I}_{\mathbf{n}-\mathbf{1}}$, with $\mathbf{1}_{\mathbf{n}} \in \mathbb{R}^{n}$ being the vector whose components are all 1 . Parameters $T>0$ and $\mu \in(0,1]$ characterize the $Q o S$ of the inter-vehicle communication network, and are an integral measure of the connectivity of graph $\mathcal{G}(t)$.

Assumption 9 Upon reception of the updated value of $\dot{x}_{R}(t)$ after a rescheduling event, the ith vehicle transmits $\dot{x}_{R}(t)$ continuously and with no delays for a limited period of time $T$ to other vehicles in the fleet.

Given the QoS for the inter-vehicle communication network specified in Assumption8, Assumption 9 guarantees that all vehicles receive the updated value of the reference rate. Considering Assumptions 1 through 9 the control objective is to design a distributed protocol that solves the following consensus problem:

$$
\begin{array}{rlr}
x_{i}(t)-x_{j}(t) \stackrel{t \rightarrow \infty}{\longrightarrow}\left[-\frac{\Delta_{c}}{2}, \frac{\Delta_{c}}{2}\right], & \forall i, j \in \mathcal{I}, \\
x_{i}(t)-x_{R}(t) \stackrel{t \rightarrow \infty}{\longrightarrow}\left[-\frac{\Delta_{a}}{2}, \frac{\Delta_{a}}{2}\right], & \forall i \in \mathcal{I}_{\ell}, \\
\dot{x}_{i}(t)-\dot{x}_{R}(t) \stackrel{t \rightarrow \infty}{\longrightarrow} 0, & \forall i \in \mathcal{I},
\end{array}
$$

where $\Delta_{c}, \Delta_{a} \geq 0$ are the width of the coordination window and the arrival window, respectively. Equation (6a) enforces the coordination constraints among the UxSs in the fleet. The leftmost term represents the inter-vehicle coordination error (ICE). Equation (6b) imposes temporal specifications on the leaders. In this case, the leftmost term represents the vehicle temporal error (VTE). Finally, Equation (6c) ensures that the mission develops at the desired rate. The leftmost term in this equation is the vehicle rate error (VRE). The next section provides a deeper insight into Equation(6) through a simple example.

\section{A. Physical Interpretation of the Consensus Problem}

Figure 2 provides a particular representation of the consensus problem defined in Equation (6). For illustrative purposes, Figure 2 shows a formation pattern for a fleet of three vehicles, the reference agent, the leader, and the follower, along with their corresponding paths depicted by dashed blue lines. All the vehicles are moving rightward, and the faded circles represent the position of the vehicles in past instances. In this particular scenario the reference agent is a real vehicle and not a virtual entity. Note, however, that the cooperative framework this paper builds upor ${ }^{12}$ does not necessarily lead to swarming behaviors.

Since the reference agent runs independently of all other vehicles, the time evolution along its path is exclusively determined by the reference rate $\dot{x}_{R}(t)$ and the desired speed profile $v_{d, R}(t)$, computed during the trajectory generation phase $\frac{23}{23}$ As a result, each point along the path of the reference agent has a fixed temporal specification, as highlighted in Figure 2. It is remarked that $\dot{x}_{R}(t)=1$ or $\dot{x}_{i}(t)=1$ for all $t$ does not imply that the speed profile of the corresponding vehicle is constant. In fact, it indicates that the UxS in question is commanded to strictly follow the desired speed profile as computed in the trajectory generation phase. Now, lets assume that after a rescheduling event the reference rate is set to $\dot{x}_{R}(t)=1.2$. In this case, the mission would evolve $20 \%$ faster than initially planned. Accordingly, the commanded speed will be the desired speed profiles scaled by 1.2 . 


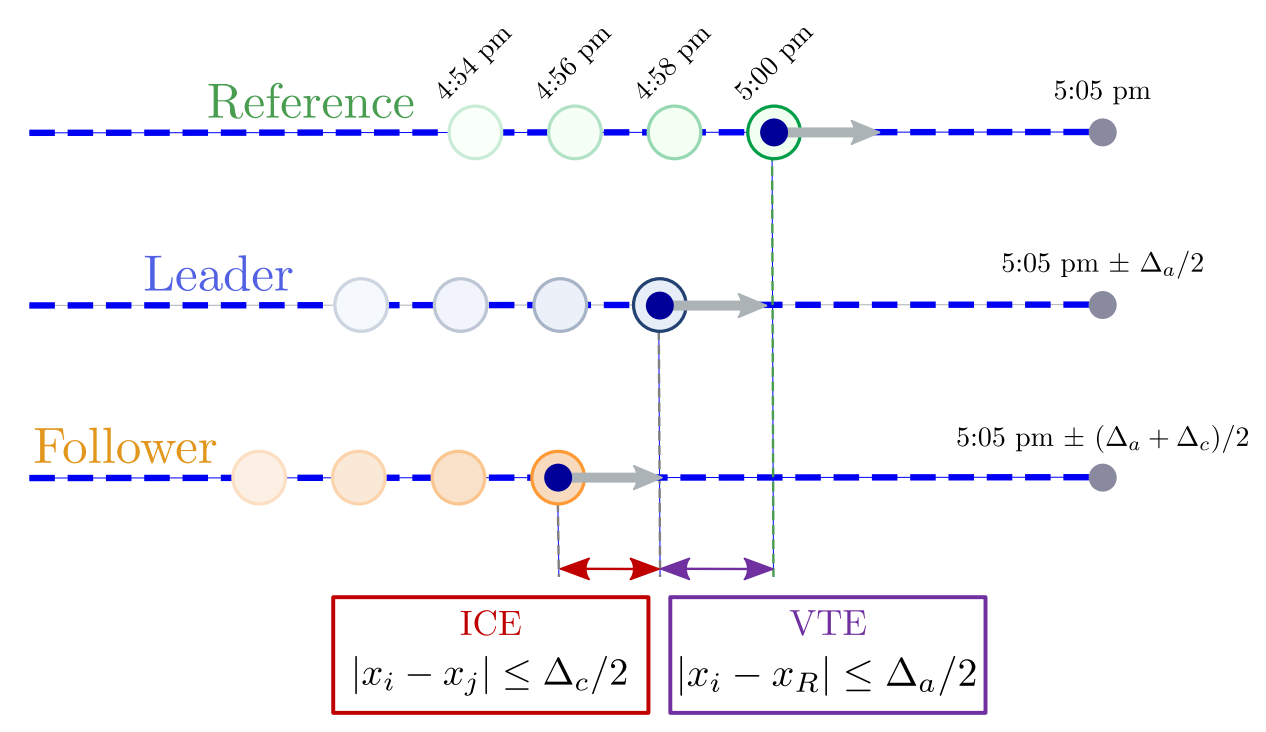

Figure 2: Illustrative example of the consensus problem.

The end-time specifications for the vehicles are shown above the gray circles that mark the end of each path. Again, for illustrative purposes, the nominal time of arrival to the final destination is 5:05 pm for all vehicles. However, this cooperative framework allows the specification of different arrival times for each of the vehicles. Further details on how to impose sequential arrival times are described in previous work ${ }^{24}$ It is also noted that this framework allows the specification of intermediate and end-time temporal constraints, which can be of interest in multi-objective missions.

Contrary to the reference agent, the leader and the follower depicted in Figure 2 exploit the information shared over the networks to achieve coordination and satisfy their temporal constraints. Note that these UxSs have, to some extent, a degree of freedom to move around a bounded neighborhood of the reference agent, defined by $\Delta_{c}$ and $\Delta_{a}$. From Figure 2 it becomes clear that $\Delta_{c}$ is a temporal measure of the allowable discoordination among all the vehicles in $\mathcal{I}$, while $\Delta_{a}$ is the leeway given to the leaders to satisfy their temporal specifications. Additionally, it can be inferred from Figure2 that temporal constrains are not necessarily the same for the leaders and the followers. In fact, Equations (6a) and (6b) lead to

$$
x_{i}(t)-x_{R}(t) \stackrel{t \rightarrow \infty}{\longrightarrow}\left[-\frac{\Delta_{a}+\Delta_{i} 00 c}{2}, \frac{\Delta_{a}+\Delta_{c}}{2}\right], \quad \forall i \in \mathcal{I}_{f} .
$$

Since followers have no knowledge of the reference state, see Figure 1 they learn their temporal specifications through coordination with the leaders. As a result, the margin of error on the temporal specifications of the followers also depends on the width of the coordination window $\Delta_{c}$. This provides the opportunity to impose a dual behavior on the temporal constraints using the vehicle hierarchy. This can be advantageous in some mission scenarios, as illustrated in the next section.

\section{Time-Coordination Strategies}

In this section, the possible values of $\Delta_{c}$ and $\Delta_{a}$ are utilized to define several types of coordination and temporal specification constraints. To emphasize the usability of the resulting time-coordination strategies, we provide real-world examples where these strategies can be used to automate procedures that are often controlled by human operators. Depending on the value of the coordination window $\Delta_{c}$, we define the following coordination types:

- Stringent coordination $\left(\Delta_{c}=0\right)$ : this strategy forces the vehicles to strictly observe their coordination constraints, and drives the ICE to zero. This type of coordination is typical of scenarios where maintaining the desired relative position among vehicles is crucial, see examples depicted in Figures 3a, 4a and 5a, Stringent coordination can be used as a tool to ensure vehicles do not violate the minimum safety distance separation when assigned time-deconflicted trajectories. 
- Weak coordination $\left(\Delta_{c}>0\right)$ : defines an allowable margin of error in the coordination among vehicles, and ensures that the ICE converges to a desired neighborhood of zero. Naturally, weak coordination is a relaxation of stringent coordination specifications. Weak coordination constraints are common in mission scenarios where coordination is still utilized to ensure inter-vehicle separation. However, in this case time-deconflicted trajectories must be computed such that a bounded error in the coordination among vehicles does not jeopardize the integrity of the UxSs. Examples shown in Figures $3 \mathrm{~b}, 4 \mathrm{~b}$, and $5 \mathrm{~b}$ correspond to scenarios where weak coordination is used.

Similarly, based on the possible values of the arrival window $\Delta_{a}$, we define the following types of temporal constraints:

- Unenforced temporal constraints $\left(\Delta_{a}\right.$ undefined): do not impose any temporal specifications on the UxSs. Since $\Delta_{a}$ is undefined, Equations (6b) and (7b) are not defined either. Note that, in this case, the function of the reference agent is limited to determining the rate of the mission, and it does not need to share the reference state with the leaders in the fleet. This type of temporal constraint is used in missions that do not require the specification of an arrival time at any point along the path of the vehicles. In scenarios with unenforced temporal constraints, the reference agent is typically a virtual entity. Next, we introduce two examples that utilize these types of constraints.

Figure 3 a presents a scenario that combines stringent coordination with unenforced temporal constraints. Coordinated maneuvers performed by the US Navy Blue Angels resulting in a specific flight formation pattern are shown in this figure. This type of exercises require that the aircraft meet desired relative position specifications with extreme precision due to the close proximity among aircraft. This can be translated into our framework as stringent coordination. At the same time, the vehicles depicted in Figure $3 \mathrm{a}$ are not subject to temporal specifications, since the aircraft are not required to arrive to any specific location at any specific time. Consequently, this particular scenario could be automated using stringent coordination and unenforced temporal constraints.

Figure $3 \mathrm{~b}$ depicts a coordinated search and rescue operation in a difficult-access mountainous area. The trajectories in blue and orange show two UAVs take off, fly a Boustrophedon-like pattern, and land at the same location they took off. The red dots indicate the location of the UAVs along their trajectories at different instances. In this case, cooperation among vehicles is leveraged to cover a larger area in a smaller amount of time. This is of especial importance in life-threatening scenarios, where prompt assessment and localization of victims significantly increases survival rates. Note that the trajectories are not spatially deconflicted, since the paths overlap at the points marked by a black cross. Therefore, in the absence of coordination the vehicles may coincide at these points, especially in the presence of external disturbances that may affect the UAVs differently, such as wind gusts. Additionally, it is often desired that the sensing regions of the vehicles slightly overlap, which also requires some sort of coordination. Notice, however that strict coordination is not completely necessary since the vehicles can deviate along their nominal trajectories to some extent without the risk of collision. Again, this scenario does not impose any temporal specifications on the UAVs since they are not required to fly above any particular point at a specific time. As a result, this mission could be implemented using weak coordination and unenforced temporal constraints. More importantly, with the adequate sensing payload, this type of missions could be automated and performed at night, when conventional manned search and rescue operations often need to halt.

- Tight temporal constraints $\left(\Delta_{a}=0\right)$ : force the leaders to strictly observe their temporal specifications. This type of temporal constraint is characteristic of missions that require the UxS to be at specific points along their paths at a particular moment in time. These points can be one or more intermediate points, as well as the location of the final destination. Again, we present two scenarios that illustrate how these strategies can be used in real-world applications.

Calibration and validation of satellite data is a representative example that combines stringent coordination with strict temporal constraints. In such experiments, one or more aircraft are tasked to fly cooperatively to collect data that will later be compared to the information obtained by the satellite. Often, the satellite over-pass and the aircraft measurements must take place simultaneously, since the variables measured (temperature, humidity, ice thickness, concentration of specific pollutants...) may vary with time. Due to the cost and complexity of orbital maneuvers, the satellite does not attempt coordination with the fleet of aircraft and acts as the reference agent. Conversely, aircraft coordinate 


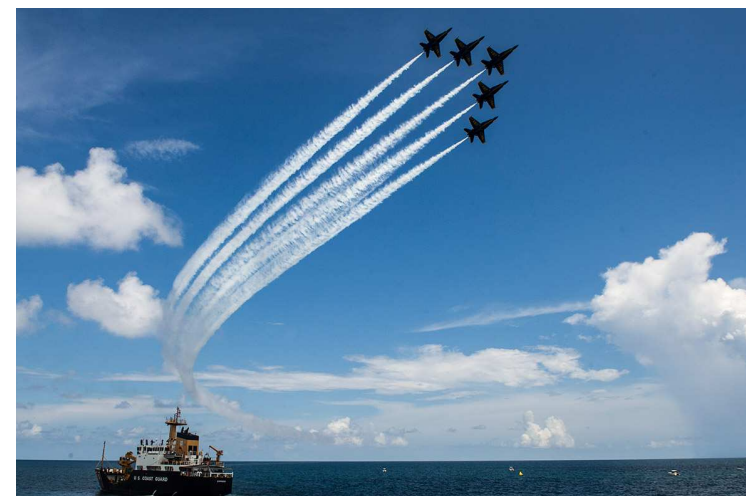

(a) Coordinated maneuvers at an airshow, stringent coordination (Image is courtesy of U.S. Navy Blue Angels).

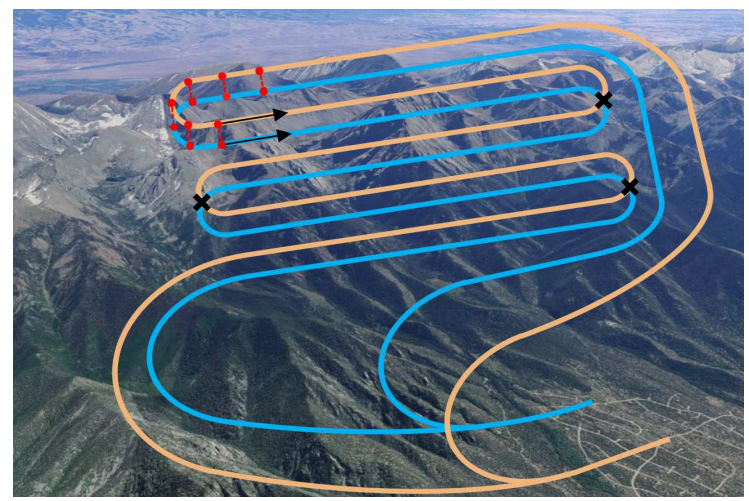

(b) Coordinated search and rescue operation, weak coordination.

Figure 3: Mission scenarios with unenforced temporal constraints.

with each other and attempt to meet the temporal and spatial specifications determined by the satellite's orbit. One example of such missions is NASA's IceBridge program, which performed low-altitude flights to calibrate the radar altimeter onboard the CryoSat-2, a European Space Agency (ESA) environmental research satellite. Figure 4a shows the coordinated trajectories of a DC-8 in yellow, and the CryoSat- 2 in orange, along with the times of the satellite over-pass. In this case, the aircraft and the satellite had to be both spatially and temporally coordinated to achieve the desired calibration accuracy, and avoid bias in the measurements due to the drifting of the sea ice pack.

Figure $4 \mathrm{~b}$ shows a fleet of vessels engaged in coordinated maneuvers. Meanwhile, three aircraft are tasked to land on the aircraft carrier highlighted in orange. Note that the vessels do not require strict coordination among themselves, since small deviations in their formation will not lead to collision. On the other hand, the aircraft must precisely meet the spatial and temporal specifications defined by the aircraft carrier. Since the carrier is the largest and most expensive vehicle to operate, it behaves as the reference agent. In order to achieve the aforementioned collective behavior, the aircraft are elected as leaders, the remaining vessels are the followers, and weak coordination with tight temporal constraints is the time-coordination strategy of choice.

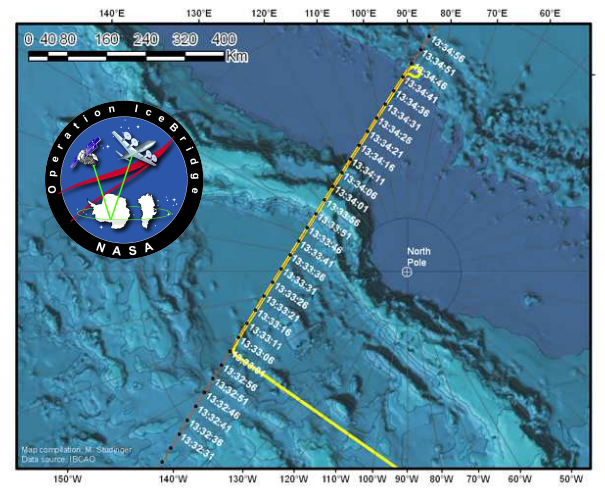

(a) Validation and calibration of satellite data, stringent coordination (Image is courtesy of Michael Studinger,27 adapted by Javier Puig-Navarro).

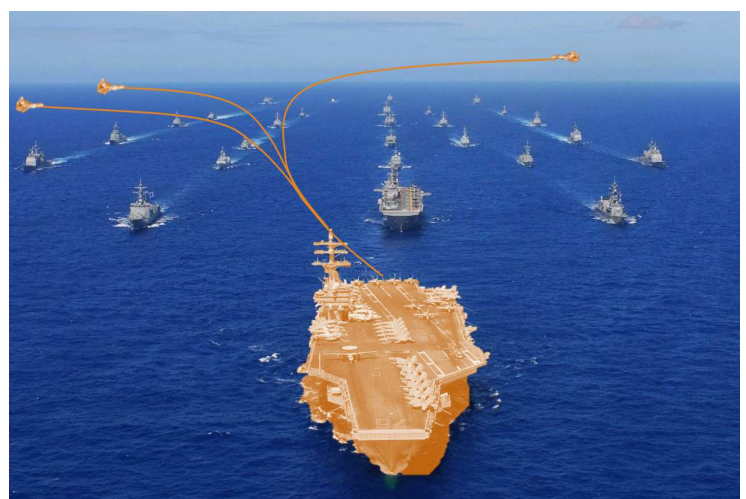

(b) Coordinated sea-air maneuvers, weak coordination (Image is courtesy of U.S. Navy, by Dylan McCord, adapted by Javier Puig-Navarro).

Figure 4: Mission scenarios with strict temporal constraints.

- Loose temporal constraints $\left(\Delta_{a}>0\right)$ : provide the leaders and consequently the followers, see Equation (7), with an allowable margin of error in their temporal specifications. Weak temporal constraints must be imposed in missions that require the vehicles to reach one or more points along their paths within a temporal window.

Figure 5a shows a coordinated aerial refueling maneuver involving two Tornado GR4 and a Boeing 
KC-135R Stratotanker. Generally, a refueling exercise is not the end goal of a mission, but it is often a needed intermediate phase. Let us assume that the two GR4 Tornado are tasked to arrive to their final destination within an arrival window, defined about a nominal time of arrival. Due to the close proximity nature of aerial refueling operations, the tanker and the two Tornado need to satisfy strict relative position constraints. Additionally, these aircraft must arrive to their final destination within a pre-defined temporal slot. As a result, this scenario could be automated using stringent coordination, and loose absolute temporal constraints.

Figure $5 \mathrm{~b}$ depicts an auto-landing scenario involving five autonomous vehicles. Each vehicle is requested to follow its assigned trajectories and land on the runway within a predefined arrival window. Figure $5 \mathrm{c}$ shows the nominal arrival time and desired arrival window for each of the vehicles. The trajectories assigned to the UAVs are time-deconflicted, and therefore require that vehicles coordinate to ensure safe inter-vehicle separation. Thus, this auto-landing scenario could be implemented using weak coordination and loose temporal constraints.

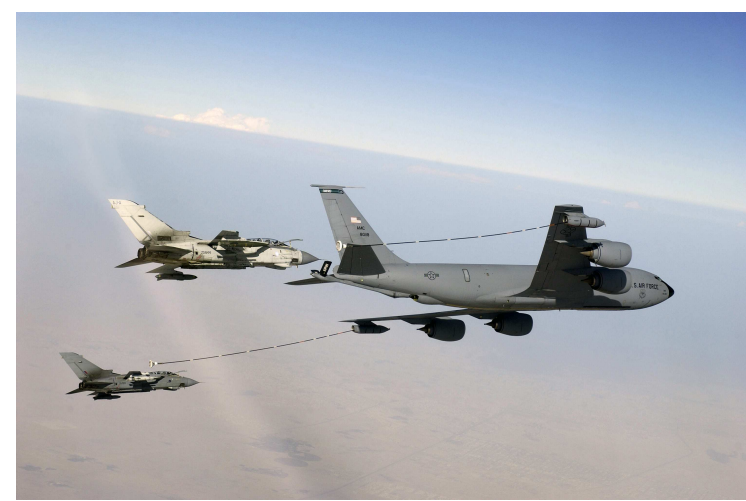

(a) Coordinated aerial refueling, stringent coordination (Image is courtesy of U.S. Air Force, by Suzanne M. Jenkins).

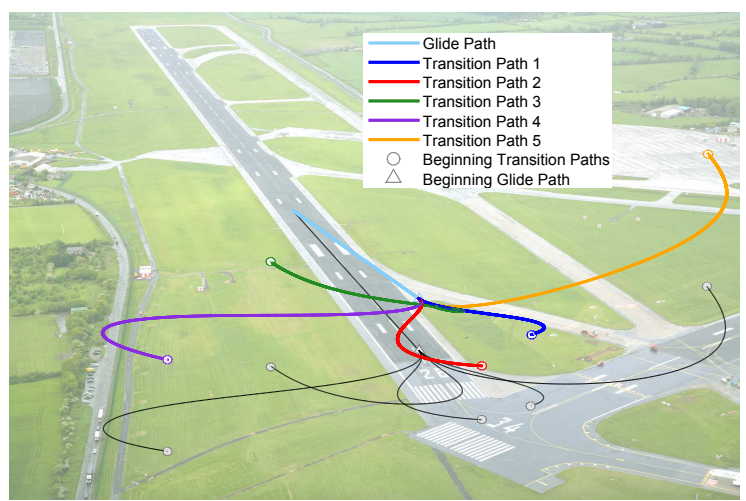

(b) Coordinated auto-landing scenario, weak coordination.

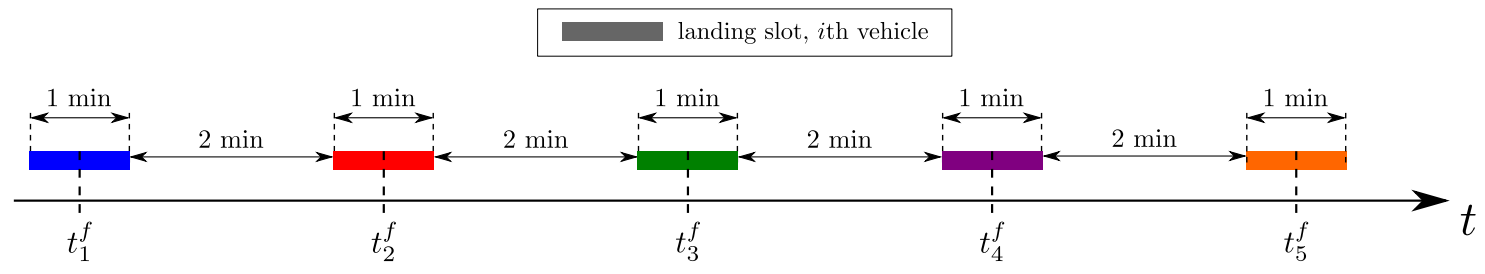

(c) Assigned landing slots for the five UAVs in the coordinated auto-landing scenario.

Figure 5: Mission scenarios with loose temporal constraints.

The combination of coordination constraints and temporal specifications introduced above defines six different time-coordination strategies, summarized in Table 1. Nonetheless, there exist scenarios with coordination and temporal requirements that are not captured by Table 1 As an example, consider a slight modification of the mission shown in Figure 4b. Figure 6 shows the same fleet of vessels engaged in coordinated maneuvers. However, three aircraft are tasked to land on the aircraft carrier highlighted in orange, while other three aircraft have to land on the carrier highlighted in green. One may choose to maintain weak coordination with tight temporal constraints, create a virtual reference agent, and elect the two carriers and six aircraft as leaders. While this may solve the problem, the degree of freedom that allowed a bounded discoordination between carriers has been eliminated. More importantly, this option would force all aircraft to precisely coordinate with each other, which is also unnecessary. As a result, we would be over-constraining the fleet. This often leads to larger control inputs, and hence larger speed corrections that result in higher fuel consumption. If one wishes to allow bounded discoordination between the carriers and among orange and green aircraft, the only solution is to define agent-specific coordination behaviors. We will refer to these constraints as individually tailored time-coordination constraints. These problems cannot be formulated as in Equation [6] Consequently, the development of distributed coordination control laws 
for individually tailored time-coordination problems is beyond the scope of this paper. Next, we provide distributed coordination control laws for the time-coordination strategies in Table 1

Table 1: Time-coordination strategies.

\begin{tabular}{|c|c|c|c|}
\hline & \multicolumn{2}{|c|}{ Coordination } \\
\hline & & Stringent & Weak \\
\hline \multirow{3}{*}{ 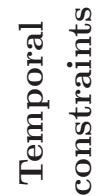 } & Unenforced & $-\quad, \Delta_{c}=0$ & $-\quad, \Delta_{c}>0$ \\
\hline & Tight & $\Delta_{a}=0, \Delta_{c}=0$ & $\Delta_{a}=0, \Delta_{c}>0$ \\
\hline & Loose & $\Delta_{a}>0, \Delta_{c}=0$ & $\Delta_{a}>0, \Delta_{c}>0$ \\
\hline
\end{tabular}

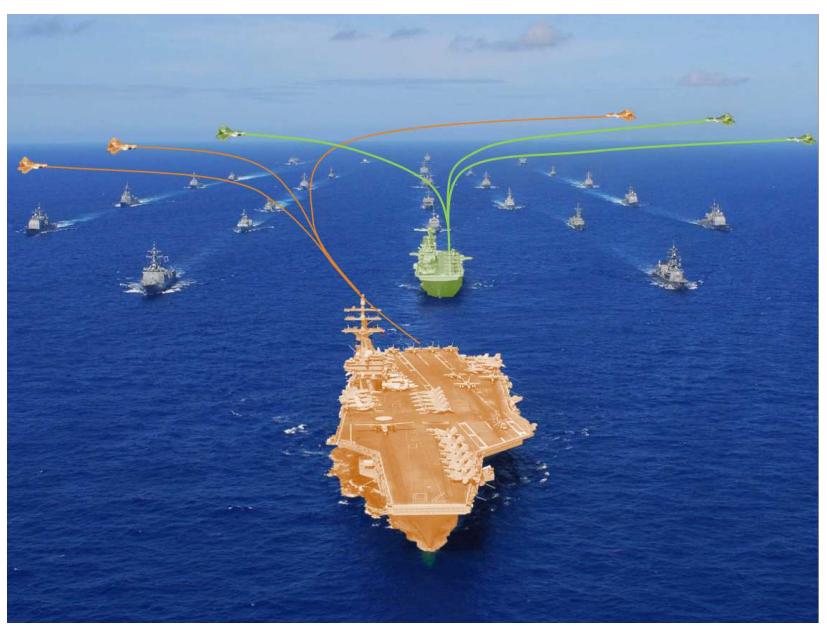

Figure 6: Mission scenario with individually tailored time-coordination constraints (Image is courtesy of U.S. Navy, by Dylan McCord, adapted by Javier Puig-Navarro).

\section{Distributed Control Law}

To solve the consensus problem defined in Equation (6), we propose the following distributed coordination control law:

$$
\begin{aligned}
& \text { Leaders }\left\{\begin{aligned}
u_{i}(t)=-k_{P} \sum_{j \in \mathcal{N}_{i}} \omega_{c_{i, j}}\left(x_{i}-x_{j}\right)-k_{P_{R}} \omega_{R_{i}} \ell_{R_{i}}\left(x_{i}-x_{R}\right)+\chi_{i} \\
\dot{\chi}_{i}(t)=-k_{I} \sum_{j \in \mathcal{N}_{i}} \omega_{c_{i, j}}\left(x_{i}-x_{j}\right)-k_{I_{R}} \omega_{R_{i}} \ell_{R_{i}}\left(x_{i}-x_{R}\right)+\alpha_{i}\left(\dot{x}_{R}-\chi_{i}\right), \quad i \in \mathcal{I}_{\ell} \\
\chi_{i}(0)=\chi_{i_{0}}
\end{aligned}\right. \\
& \text { Followers }\left\{\begin{aligned}
u_{i}(t)=-k_{P} \sum_{j \in \mathcal{N}_{i}} \omega_{c_{i, j}}\left(x_{i}-x_{j}\right)+\chi_{i} & , \quad i \in \mathcal{I}_{f} \\
\dot{\chi}_{i}(t) & =-k_{I} \sum_{j \in \mathcal{N}_{i}} \omega_{c_{i, j}}\left(x_{i}-x_{j}\right)+\alpha_{i}\left(\dot{x}_{R}-\chi_{i}\right) \\
\chi_{i}(0) & =\chi_{i_{0}}
\end{aligned}\right.
\end{aligned}
$$

where $k_{P}, k_{I}, k_{P_{R}}, k_{I_{R}}>0$ are control gains; $\omega_{c_{i, j}}(t)$ is the coordination link weight associated with vehicles $i$ and $j ; \omega_{R_{i}}(t)$ is the reference link weight of the $i$ th leader; $\alpha_{i}(t)=1$ if $\dot{x}_{R}(t)$ is available to the $i$ th $\mathrm{UxS}$, $\alpha_{i}(t)=0$ otherwise; and $\chi_{i}(t) \in \mathbb{R}$ is the integral state of the $i$ th fleet member. The distributed coordination control law has a proportional integral structure that provides both leaders and followers with disturbance rejection capabilities. The main difference between the coordination control law for the leaders and the followers is the use of the reference state. Notice that leaders use the reference state to enforce temporal 
constraints whenever it is available through the network, namely $\ell_{R_{i}}=1$; while followers cannot use this information since it is not available to them, as illustrated in Figure 1 Next, we remark the functionality of the three distinct terms in the coordination control law

- Terms that include the coordination link weight $\omega_{c_{i, j}}(t)$ enforce coordination among vehicles.

- Terms with the reference link weight $\omega_{R_{i}}(t)$ impose temporal constraints on the leaders. Note that this term is not present in the control law of the followers. Consequently, followers learn their temporal specifications through coordination with the leaders, as previously inferred from Equation (77).

- Terms with $\dot{x}_{R}(t)$ help UxSs learn the desired rate of the mission. As a remark, in the case of stringent coordination convergence can be achieved even if $\alpha_{i}(t)=0$ for all $t$ in Equation (8b). This occurs because the integral term in the control law allows the follower to learn a new reference rate from the leaders. However, the availability of the reference rate $\dot{x}_{R}(t)$ in Equation (8b), namely $\alpha_{i}(t)=1$, improves convergence of the integral state of the followers to the desired rate of the mission.

Recall that Equation (8) is the proposed solution for all the time-coordination strategies defined in Section IV] Next, we define the link weight decision logic for each of the time-coordination strategies in Table 1.

\section{A. Link Weight Decision Logic}

The decision logic of the coordination link weights $\omega_{c_{i, j}}(t)$ defines the type of coordination constraints enforced on the UxSs. Table 2 contains the decision logic for stringent and weak coordination. From Equation (8), we know $\omega_{c_{i, j}}(t)$ is not used if $j \notin \mathcal{N}_{i}(t)$. Therefore, in such instances the value of $\omega_{c_{i, j}}(t)$ does not need to be specified. Notice that for stringent coordination the $i$ th vehicle always "listens" to the $j$ th $\mathrm{UxS}$ if the coordination state of the latter is available through network. However, for weak coordination the $i$ th vehicle only "listens" to the $j$ th UxSs if the coordination state of the latter is available through the network and is outside the coordination window, specified by $\Delta_{c}$. In order to avoid chattering, the decision logic for weak coordination is also subject to slow switching constraints. To this end, we define the switching times $t_{c_{i, j, 1}}, t_{c_{i, j, 2}}, \ldots$ and delay the switching of $\omega_{c_{i, j}}(t)$, defined in Table 2, until the following inequality is met:

$$
t_{c_{i, j, k+1}}-t_{c_{i, j, k}} \geq \tau_{d_{c}}, \quad \forall k \in \mathbb{N},
$$

where $\tau_{d_{c}}$ is the dwell time for the coordination link weights. Furthermore, it is expected that these slow switching constraints are required to prove stability of the control law for weak coordination.

Table 2: Coordination link weight decision logic.

\begin{tabular}{|c|c|}
\hline Stringent & Weak \\
\hline \hline$\omega_{c_{i, j}}(t)=1$ & $\omega_{c_{i, j}}(t)= \begin{cases}1, & \left|x_{i}(t)-x_{j}(t)\right| \geq \Delta_{c} / 2 \\
0, & \left|x_{i}(t)-x_{j}(t)\right|<\Delta_{c} / 2\end{cases}$ \\
\hline
\end{tabular}

Similarly, the decision logic of the reference link weights $\omega_{R_{i}}(t)$ defines the type of temporal constraints imposed on the fleet. Table 3 contains the decision logic for unenforced, tight, and loose temporal specifications. As shown in Equation (8), $\omega_{R_{i}}(t)$ is not utilized if $\ell_{R_{i}}(t)=0$. Consequently, the value of $\omega_{R_{i}}(t)$ does not need to be defined in such instances. Table 3 determines that for unenforced temporal constraints the leaders never "listen" to the reference state. Conversely, for tight temporal constraints leaders always "listen" to the reference state, if available through the reference-leaders communication network. Finally, for loose temporal specification leaders only listen to the reference state if available through the network and outside the arrival window. Again, to avoid chattering the decision logic for loose temporal specifications is subject to slow switching constraints. To this purpose, consider the switching times $t_{R_{i, 1}}, t_{R_{i, 2}}, \ldots$ and delay the switching of $\omega_{R_{i}}(t)$ until the following inequality is met:

$$
t_{R_{i, k+1}}-t_{R_{i, k}} \geq \tau_{d_{R}}, \quad \forall k \in \mathbb{N},
$$

where $\tau_{d_{R}}$ is the dwell time for the reference link weights. As before, it is expected that these slow switching constraints be required to prove stability of the control law for loose temporal specifications. 
Table 3: Reference link weight decision logic.

\begin{tabular}{|c|c|c|}
\hline Unenforced & Strict & Loose \\
\hline \hline$\omega_{R_{i}}(t)=0$ & $\omega_{R_{i}}(t)=1$ & $\omega_{R_{i}}(t)= \begin{cases}1, & \left|x_{i}(t)-x_{R}(t)\right| \geq \Delta_{a} / 2 \\
0, & \left|x_{i}(t)-x_{R}(t)\right|<\Delta_{a} / 2\end{cases}$ \\
\hline
\end{tabular}

\section{Simulation Scenarios}

To demonstrate the efficacy of the control law proposed in Equation (8), simulation results for the six time-coordination strategies are presented. All the simulation scenarios were run for a fleet of $n=5$ vehicles with the same initial conditions, reference-leader network topology, and inter-vehicle network topology. Vehicles 1 and 2 are designated as leaders. The initial conditions for the coordination states $x_{i}(t)$ were randomly picked from a ball in $\mathbb{R}^{n}$ of radius $r_{x}=\left\|\mathrm{x}_{0}\right\|_{2}=10 \mathrm{~s}$ centered at the origin, and with uniform probability distribution, where $\mathbf{x}_{0}:=\left[x_{1_{0}}, x_{2_{0}}, x_{3_{0}}, x_{4_{0}}, x_{5_{0}}\right]^{\top}$. The initial values for the coordination states are

$$
\mathbf{x}_{\mathbf{0}}=[-0.21,3.58,6.59,0.00,-2.86]^{\top} \text {. }
$$

Similarly, initial conditions for the integral states $\chi_{i}(t)$ were randomly picked from a ball in $\mathbb{R}^{n}$ of radius $r_{\chi}=\left\|\chi_{\mathbf{0}}\right\|_{2}=0.30 \mathrm{~s} / \mathrm{s}$ centered at $\mathbf{1}_{\mathbf{n}}$, and with uniform probability distribution, where $\chi_{\mathbf{0}}:=\left[\chi_{1_{0}}, \chi_{2_{0}}, \chi_{3_{0}}, \chi_{4_{0}}, \chi_{5_{0}}\right]^{\top}$. The initial values for the integral states are

$$
\chi_{\mathbf{0}}=[0.79,1.15,0.98,1.01,0.92]^{\top} .
$$

Initially, the reference rate is set to the nominal value. However, at $t=30 \mathrm{~s}$ a rescheduling event occurs. The time-evolution of the reference rate can be expressed as

$$
\dot{x}_{R}(t)=\left\{\begin{array}{ll}
1, & 0 \leq t<30 \\
1.2, & t \geq 30
\end{array} .\right.
$$

The inter-vehicle time-varying network topology was generated using a pseudo-random network generator that lets the user specify upper and lower bounds on the connectivity of the network $\hat{\mu}_{\min } \leq \hat{\mu}(t) \leq \hat{\mu}_{\max }$ for a given $T$, where $\hat{\mu}(t)$ is defined as follows:

$$
\hat{\mu}(t):=\frac{1}{n} \frac{1}{T} \int_{t-T}^{t} \mathbf{Q L}(\tau) \mathbf{Q}^{\top} d \tau, \quad \forall t \geq T .
$$

These parameters were set to the following values:

$$
\hat{\mu}_{\min }=0.25, \quad \hat{\mu}_{\max }=0.40, \quad T=5.00 \mathrm{~s} .
$$

The reference-leaders communication network topology was generated using a modification of the pseudorandom network topology generator. This algorithm lets the user specify upper and lower bounds on the connectivity of the network for each of the leaders $\hat{\mu}_{R, i_{\min }} \leq \hat{\mu}_{R, i}(t) \leq \hat{\mu}_{R, i_{\max }}$ for a given $T_{R}$, where $i \in \mathcal{I}_{\ell}$ and $\hat{\mu}_{R, i}(t)$ is defined as follows:

$$
\hat{\mu}_{R, i}(t):=\frac{1}{T_{R}} \int_{t-T}^{t} \ell_{R_{i}}(\tau) d \tau, \quad \forall t \geq T_{R}, \quad i \in \mathcal{I}_{\ell} .
$$

The values chosen for those parameters are

$$
\hat{\mu}_{R, i_{\min }}=0.30, \quad \hat{\mu}_{R, i_{\max }}=1.00, \quad T_{R}=5.00 \mathrm{~s}, \quad \forall i \in \mathcal{I}_{\ell} .
$$

Figures 7a and 7b show the connectivity of the inter-vehicle communication network and the reference-leaders communication network, respectively. Recall Assumptions 4 and 8 and note that for the simulation scenarios presented $\mu=0.27$ and $\mu_{R}=0.3$.

The values chosen for the control gains are

$$
k_{P}=0.20, \quad k_{I}=0.02, \quad k_{P_{R}}=0.20, \quad k_{I_{R}}=0.02 .
$$




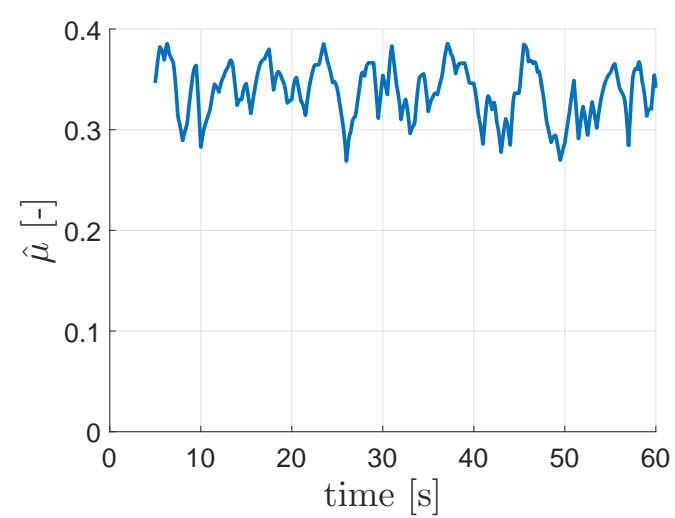

(a) Inter-vehicle communication network.

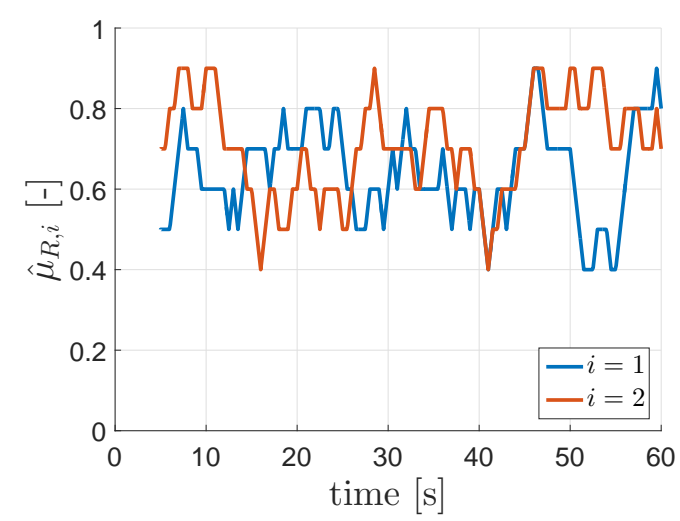

(b) Reference-leaders communication network.

Figure 7: Connectivity.

The width of the coordination window, the arrival window, and the dwell times for the link weight decision logic are set to

$$
\Delta_{c}=4.00 \mathrm{~s}, \quad \Delta_{a}=2.00 \mathrm{~s}, \quad \tau_{d_{c}}=1.00 \mathrm{~s}, \quad \tau_{d_{R}}=1.00 \mathrm{~s} .
$$

Figure 9 depicts the simulation results for unenforced temporal specifications with both stringent and weak coordination constraints. Notice that in the case of stringent coordination the ICE converges to the origin, see Figure 9a, However, for weak coordination the ICE converges to a $\Delta_{c} / 2$-neighborhood of the origin, see Figure 9b. The black dashed lines in Figures 9a and 9b indicate the control objective for the ICE. Observe that for stringent coordination the control objective is depicted by a single line; whereas for weak coordination it is represented by two dashed lines, and anything that falls between them meets the coordination specifications. Note also that convergence to the desired set occurs significantly faster for weak coordination since the fleet is asked for a smaller control effort. Figures $9 \mathrm{c}$ and $9 \mathrm{~d}$ show the VTE for stringent and weak coordination, respectively. It can be observed that the VTE of all the vehicles converges to a single bounded value in Figure 9c while in Figure 9d the VTE of each vehicle stabilizes at different values. Notice that Figures 9c and 9d do not include any dashed black lines, indicating that there are no control objectives defined in terms of the VTE, as corresponds to unenforced temporal constraints. Figures 9e and 9f show the time evolution of the integral states for stringent and weak coordination, respectively. In both cases, the integral states converge to the reference rate, even after the rescheduling event. Figures $9 \mathrm{~g}$ and $9 \mathrm{~h}$ depict the control input associated with the aforementioned coordination strategies. The non-smooth nature of the control input is the result of the switching inter-vehicle and reference-leaders network topologies. For weak coordination, Figure 9h. the switching of the coordination link weights also contributes to the nonsmoothness of the control input. There exist several techniques suitable to reduced this type of effects. In particular, the development of estimators that provide useful information about the coordination state of other vehicles while information is not received over the network can be used to smoothen the control input. Another, technique would be the use of continuous link weights that do not lead to hybrid system behaviors. Observe that, in general, the control input converges faster to the desired reference rate for weak coordination.

Figure 10 shows the simulation results for strict temporal specifications with both stringent and weak coordination. Again, stringent coordination forces all the vehicles to converge to the same coordination state, as shown in Figure 10a whereas weak coordination allows bounded disagreement in the coordination states of all vehicles, see Figure 10b Figures $10 \mathrm{c}$ and $10 \mathrm{~d}$ show the time-evolution of the VTE. The major difference with Figures $9 \mathrm{c}$ and $9 \mathrm{~d}$ is the presence of dashed black lines indicating that temporal constraints are now being actively enforced on the vehicles. Figure 10c indicates that the coordination state of all the vehicles eventually converges to the reference coordination state, as expected of stringent coordination with strict temporal constraints. For weak coordination, Figure 10d shows how the coordination state of the two leaders converges to the reference state, while the coordination states of the followers converge to and remain in a $\Delta_{c} / 2$-neighborhood of the reference state. This behavior was anticipated by Equations (7) and (6b). Figures $10 \mathrm{e}$ and $10 \mathrm{f}$ show the evolution of the integral states. Again, the integral states converge to the reference rate, even after the rescheduling event. Figures $10 \mathrm{~g}$ and $10 \mathrm{~h}$ show the time-evolution of the control 
input for stringent and weak coordination, respectively.

Figure 11 shows the simulation results for loose temporal specifications with both stringent and weak coordination constraints. Figures $11 \mathrm{c}$ and [11d show the VTE for stringent and weak coordination, respectively. Note that in Figure $11 \mathrm{c}$ the VTE converges to the same value as time progresses due to the stringent coordination constraints. Simultaneously, the VTE of all vehicles satisfies the loose temporal specifications depicted by the two black dashed lines. Figure 11d shows four black dashed lines. The region defined by the inner lines represents the temporal specifications of the leaders; whereas, the area between the two outer lines represents the temporal specifications of the followers. This is due to the fact that followers do not have access to the reference state and learn their temporal constraints through coordination with the leaders. In this particular case, the VTE of all vehicles converges to the region defined by the inner dashed black lines. Conclusions regarding the remaining subfigures are similar to the observations made for Figures 9 and 10 .

The simulation results presented in Figures 9, 10, and 11 provide numerical evidence that the control law defined in Equation (8), along with the link weight decision logic in Tables 2 and 3 , solve the consensus problem defined in Equation (6).

\section{A. The Importance of Selecting Appropriate Constraints}

Given the control law in Equation (8) and the link weight decision logic defined in Tables 2 and 3 one may question if the complexity introduced in the system as compared to previous consensus algorithms is justifiable. Specially considering that with stringent coordination and strict temporal specifications all the missions examples described in Section [V could be performed safely. Note however that this approach will constrain the systems in unnecessary ways. To answer this fundamental question, we introduce a measure of the control effort

$$
\epsilon(t)=\dot{\mathbf{x}}(t)-\dot{x}_{R}(t) \mathbf{1}_{\mathbf{n}},
$$

where $\dot{\mathbf{x}}(t)=\left[\dot{x}_{1}(t), \ldots, \dot{x}_{n}(t)\right]^{\top}$. Recall that $\dot{x}_{i}(t)$ acts as a scaling factor of the speed profile in the cooperative path-following framework that this paper builds upon! 12 Thus, $\epsilon(t)$ is related to the speed command and fuel consumption of the fleet of UxSs. Figure 8 shows the truncated $\mathcal{L}_{2}$ norm of $\epsilon(t)$ as a function of time for the six simulation scenarios represented in Figures 9, 10, and 11. The acronyms CC and TC in the legend stand for Coordination Constraints and Temporal Constraints, respectively. Observe that the control effort for weak coordination is significantly smaller than for stringent coordination. Note also that the control effort associated with unenforced temporal constraints is smaller than that of loose temporal specifications, which at the same time is smaller than that of strict temporal constraints. Consequently, if utilized appropriately the additional complexity introduced can be leveraged to reduce the collective control effort of the fleet, leading to a reduction in fuel consumption. In summary, operators with supervisory control over the cooperative framework should avoid over-constraining the system by imposing the least restrictive coordination and temporal specifications that the fleet can admit to perform the mission safely.

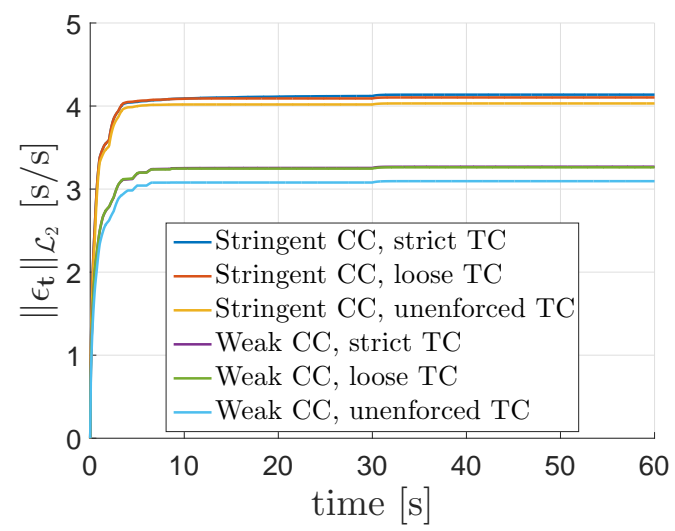

Figure 8: Truncated $\mathcal{L}_{2}$ norm of the control effort. 


\section{Conclusions}

This paper expands the spectrum of time-coordination strategies available for multi-UxSs missions. In particular, six standard time-coordination strategies that naturally result from the combination of coordination constraints and temporal specifications are proposed in this work. Real world scenarios where each strategy can be used are discussed to show the applicability of this framework. A mission scenario that requires a coordination behavior unattainable to this framework is used to illustrate the limitations of the current set of time-coordination strategies. This example is also used to introduce agent-specific coordination constraints, which will be the object of future research. A distributed control law capable of enforcing the coordination and temporal specifications discussed is formulated in this paper. The control law proposed utilizes the decision logic of a set of link weights to define the type of coordination and temporal constraints imposed on the vehicles. Simulation results for the six time-coordination strategies provide numerical evidence of the efficacy of the proposed control law. Future efforts will include the formal derivation of conditions under which the proposed control law is stable. The analysis of the simulation results highlights the importance of imposing the least restrictive set of constraints on the vehicles. By doing so, the collective control effort will be reduced, leading to a decrease in fuel consumption.

\section{References}

${ }^{1}$ Cokelet, E. D., Meinig, C., Lawrence-Slavas, N., Stabeno, P. J., Mordy, C. W., Tabisola, H. M., Jenkins, R., and Cross, J. N., "The Use of Saildrones to Examine Spring Conditions in the Bering Sea," Oceans, Washington, DC, October 2015, pp. $1-7$.

${ }^{2}$ Durban, J. W., Moore, M. J., Chiang, G., Hickmott, L. S., Bocconcelli, A., Howes, G., Bahamonde, P. A., Perryman, W. L., and LeRoi, D. J., "Photogrammetry of Blue Whales with an Unmanned Hexacopter," Marine Mammal Science, Vol. 32, No. 4, October 2016, pp. 1510-1515.

${ }^{3}$ Samiappan, S., Turnage, G., Hathcock, L., Casagrande, L., Stinson, P., and Moorhead, R., "Using Unmanned Aerial Vehicles for High-resolution Remote Sensing to Map Invasive Phragmites Australis in Coastal Wetlands," International Journal of Remote Sensing, Vol. 38, No. 8-10, October 2016, pp. 2199-2217.

${ }^{4}$ Colomina, I. and Molina, P., "Unmanned Aerial Systems for Photogrammetry and Remote Sensing: A Review," Journal of Photogrammetry and Remote Sensing, Vol. 92, June 2014, pp. 79-97.

${ }^{5}$ Gomez, C. and Purdie, H., "UAV-based Photogrammetry and Geocomputing for Hazards and Disaster Risk Monitoring - A Review," Geoenvironmental Disasters, Vol. 3, No. 1, November 2016, pp. 1-11.

${ }^{6}$ Yeong, S. P., King, L. M., and Dol, S. S., "A Review on Marine Search and Rescue Operations Using Unmanned Aerial Vehicles," International Journal of Mechanical, Aerospace, Industrial, Mechatronic and Manufacturing Engineering, Vol. 9, No. 2, November 2015, pp. 396-399.

${ }^{7}$ Waharte, S. and Trigoni, N., "Supporting Search and Rescue Operations with UAVs," International Conference on Emerging Security Technologies, Canterbury, United Kingdom, September 2010, pp. 142-147.

${ }^{8}$ Nex, F. and Remondino, F., "UAV for 3D Mapping Applications: a Review," Applied Geomatics, Vol. 6, No. 1, November 2013, pp. 1-15.

${ }^{9}$ Gatziolis, D., Lienard, J. F., Vogs, A., and Strigul, N. S., "3D Tree Dimensionality Assessment Using Photogrammetry and Small Unmanned Aerial Vehicles," PLOS ONE, Vol. 10, No. 9, September 2015, pp. 1-21.

10 "Concept of operations for the Next Generation Air Transportation System," Version 3.2, Joint Planning and Development Office, Washington, DC, September 2010, http://www.dtic.mil/dtic/tr/fulltext/u2/a535795.pdf.

${ }^{11}$ Xargay, E., Kaminer, I., Pascoal, A. M., Hovakimyan, N., Dobrokhodov, V., Cichella, V., Aguiar, A. P., and Ghabcheloo, R., "Time-Critical Cooperative Path Following of Multiple Unmanned Aerial Vehicless over Time-Varying Networks," Journal of Guidance, Control and Dynamics, Vol. 36, No. 2, March-April 2013, pp. 499-516.

${ }^{12}$ Xargay, E., Time-Critical Cooperative Path-Following Control of Multiple Unmanned Aerial Vehicles, Ph.D. thesis, University of Illinois at Urbana-Champaign, Urbana, Illinois, 2013.

${ }^{13}$ Xargay, E., Choe, R., Hovakimyan, N., and Kaminer, I., "Multi-leader coordination algorithm for networks with switching topology and quantized information," Automatica, Vol. 50, No. 3, March 2014, pp. 841-851.

${ }^{14}$ Puig-Navarro, J., Xargay, E., Choe, R., and Hovakimyan, N., "Time-Critical Coordination of Multiple UAVs with Absolute Temporal Constraints," AIAA Guidance, Navigation and Control Conference, Kissimmee, FL, January 2015, AIAA 20150595.

${ }^{15}$ McLain, T. W. and Beard, R. W., "Coordination Variables, Coordination Functions, and Cooperative Timing Missions," Journal of Guidance, Control and Dynamics, Vol. 28, No. 1, January-February 2005, pp. 150-161.

${ }^{16}$ Ghabcheloo, R., Pascoal, A. M., Silvestre, C., and Kaminer, I., "Coordinated Path Following Control of Multiple Wheeled Robots Using Linearization Techniques," International Journal of Systems Science, Vol. 37, No. 6, May 2005, pp. 399-414.

${ }^{17}$ Skjetne, R., Moi, S., and Fossen, T. I., "Nonlinear Formation Control of Marine Craft," IEEE Conference on Decision and Control, Vol. 2, Las Vegas, NV, December 2002, pp. 1699-1704.

${ }^{18}$ Ihle, I.-A. F., Coordinated Control of Marine Craft, Ph.D. thesis, Norwegian University of Science and Technology, Trondheim, Norway, September 2006.

${ }^{19}$ Peyami, E. and Fossen, T. I., "Motion Control of Marine Craft Using Virtual Positional and Velocity Constraints," International Conference on Control and Automation, Santiago, Chile, December 2011, pp. 410-416. 
${ }^{20}$ Kaminer, I., Yakimenko, O. A., Pascoal, A. M., and Ghabcheloo, R., "Path Generation, Path Following and Coordinated Control for Time-Critical Missions of Multiple UAVs," American Control Conference, Mineapolis, MN, June 2006, pp. 49064913.

${ }^{21}$ Xargay, E., Choe, R., Hovakimyan, N., and Kaminer, I., "Convergence of a PI Coordination Protocol in Networks with Switching Topology and Quantized Measurements," IEEE Conference on Decision and Control, Maui, HI, December 2012, pp. $6107-6112$.

${ }^{22}$ Cichella, V., Kaminer, I., Dobrokhodov, V., Xargay, E., Choe, R., Hovakimyan, N., Aguiar, A. P., and Pascoal, A., "Cooperative Path-Following Control of Multiple Multirotors Over Faulty Networks," IEEE Transactions on Automation Science and Engineering, Vol. 12, No. 3, July 2015, pp. 945-957.

${ }^{23}$ Choe, R., Puig-Navarro, J., Cichella, V., Xargay, E., and Hovakimyan, N., "Cooperative Trajectory Generation Using Pythagorean Hodograph Bézier Curves," Journal of Guidance, Control and Dynamics, Vol. 39, No. 8, August 2016, pp. 17441763.

${ }^{24}$ Choe, R., Distributed Cooperative Trajectory Generation for Multiple Autonomous Vehicles Using Pythagorean Hodograph Bézier Curves, Ph.D. thesis, University of Illinois at Urbana-Champaign, Urbana, Illinois, 2017.

${ }^{25}$ Bachman, G., Narici, L., and Beckenstein, E., Fourier and Wavelet Analysis, Springer, New York, USA, 2000.

${ }^{26}$ Arcak, M., "Passivity as a Design Tool for Group Coordination," IEEE Transactions on Automatic Control, Vol. 52, No. 8, August 2007, pp. 1380-1390.

${ }^{27}$ Studinger, M., "Operation IceBridge - Personal Blog," http://blogs.nasa.gov/icebridge/tag/studinger/, [Online; accessed 28 April 2016]. 


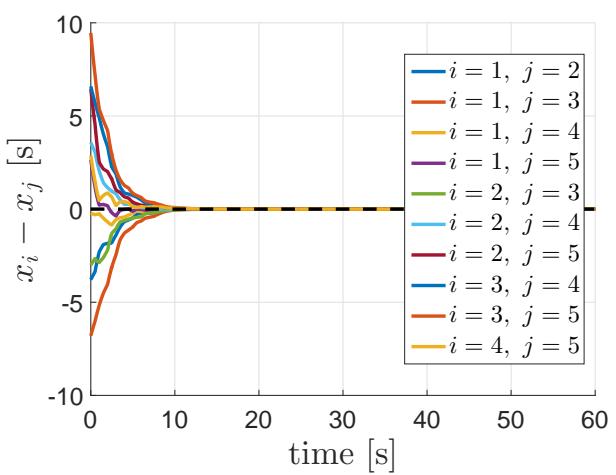

(a) Inter-vehicle coordination error, stringent coordination.

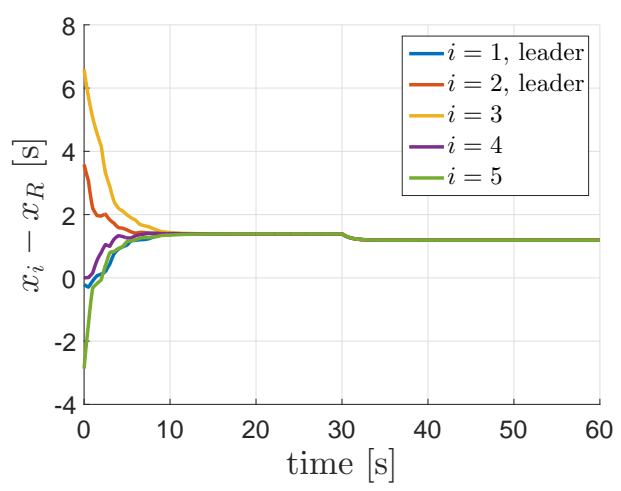

(c) Reference-vehicle coordination error, stringent coordination.

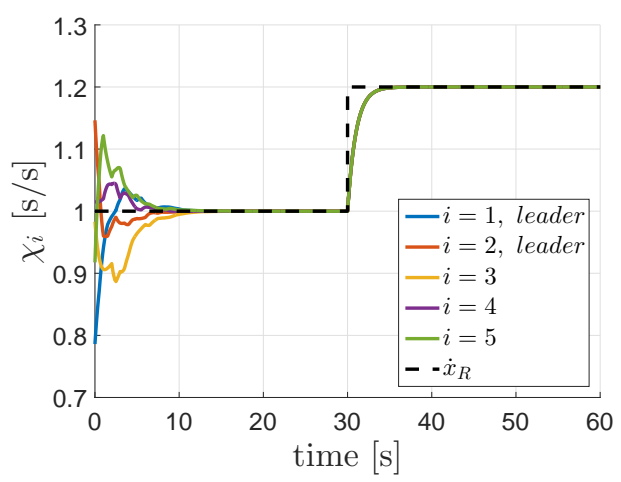

(e) Integral states, stringent coordination.

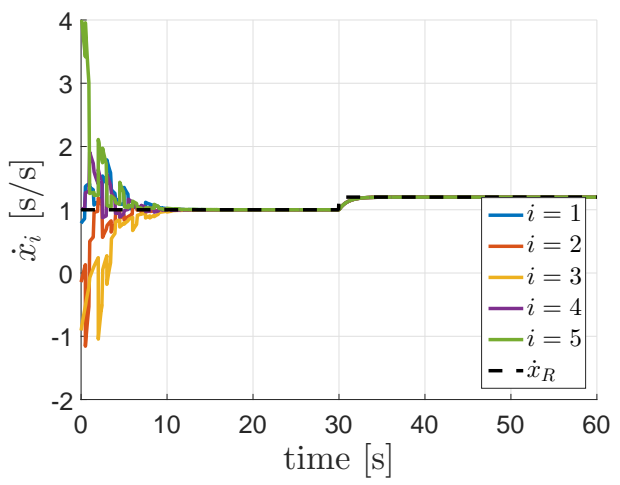

(g) Coordination-state rates, stringent coordination.

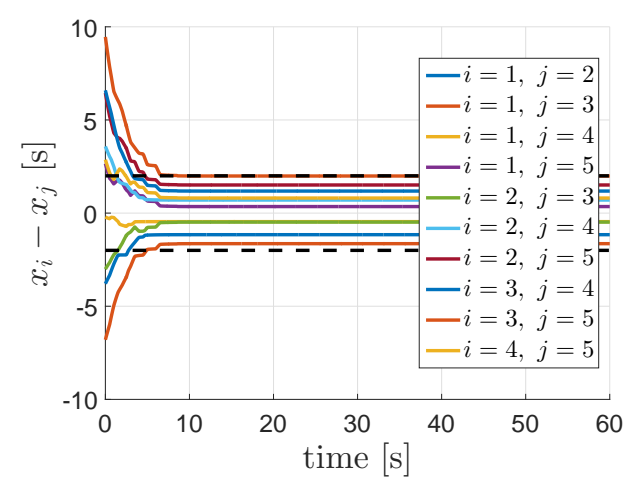

(b) Inter-vehicle coordination error, weak coordination.

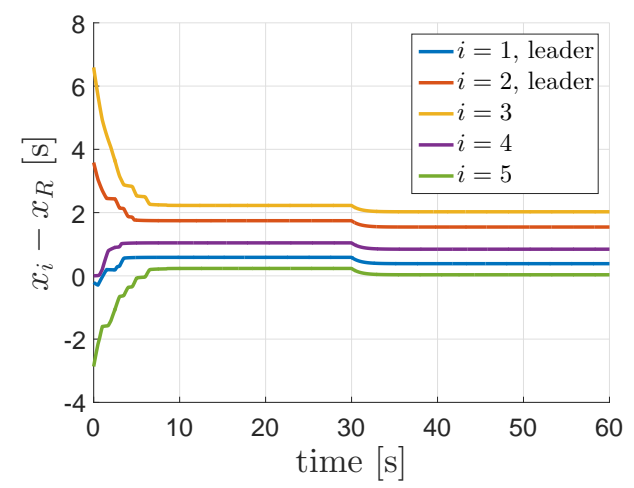

(d) Reference-vehicle coordination error, weak coordination.

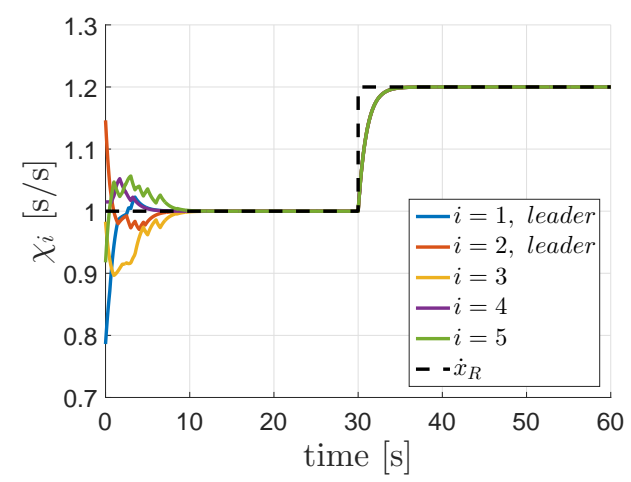

(f) Integral states, weak coordination.

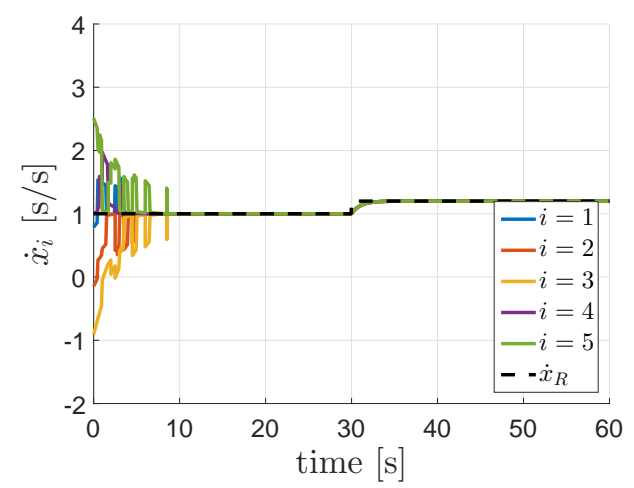

(h) Coordination-state rates, weak coordination

Figure 9: Unenforced temporal constraints. 


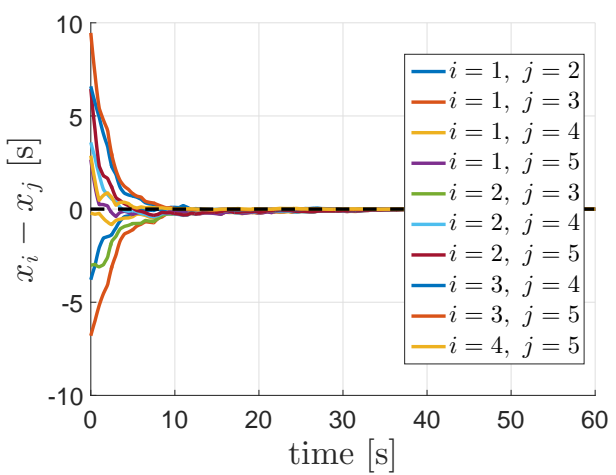

(a) Inter-vehicle coordination error, stringent coordination.



(c) Reference-vehicle coordination error, stringent coordination.

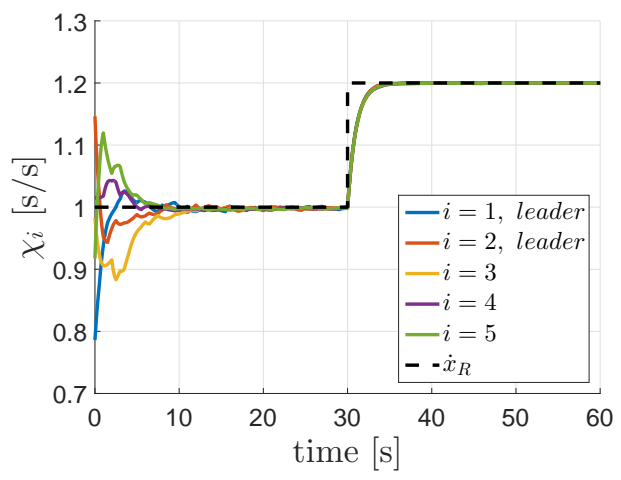

(e) Integral states, stringent coordination.

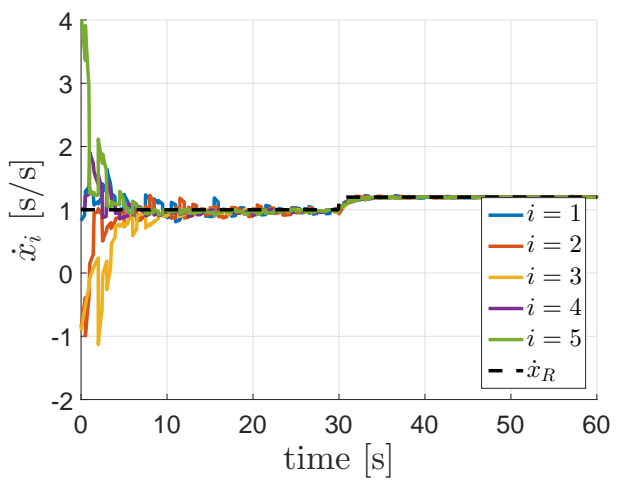

(g) Coordination-state rates, stringent coordination.

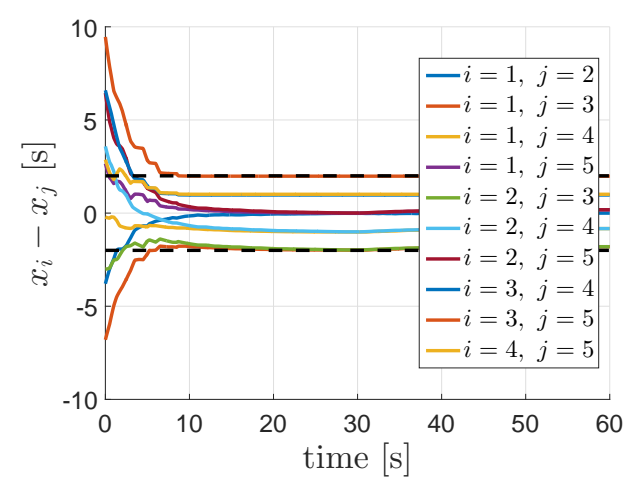

(b) Inter-vehicle coordination error, weak coordination.

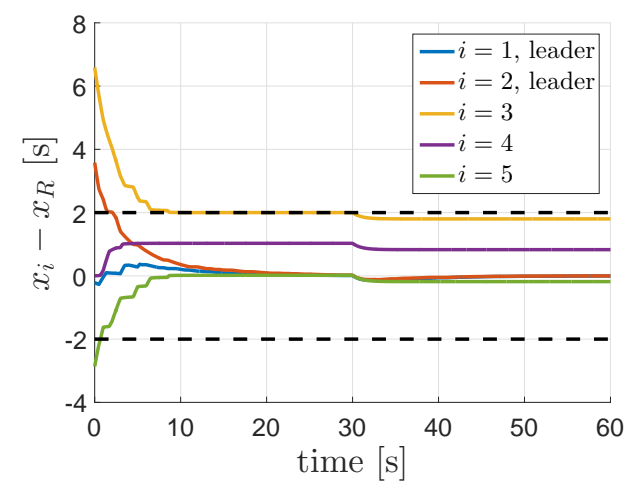

(d) Reference-vehicle coordination error, weak coordination.

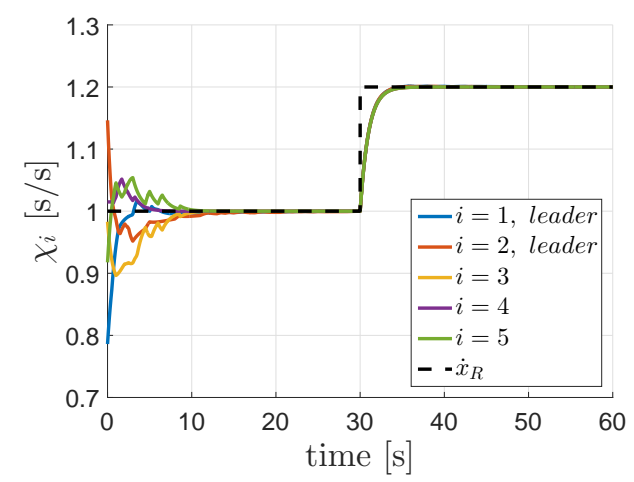

(f) Integral states, weak coordination.

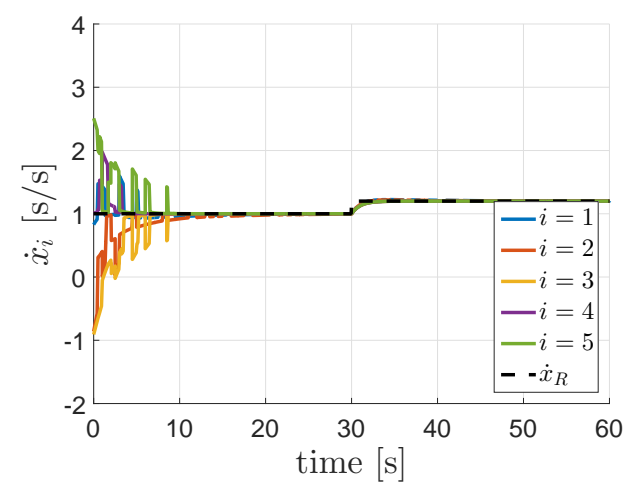

(h) Coordination-state rates, weak coordination

Figure 10: Strict temporal constraints. 


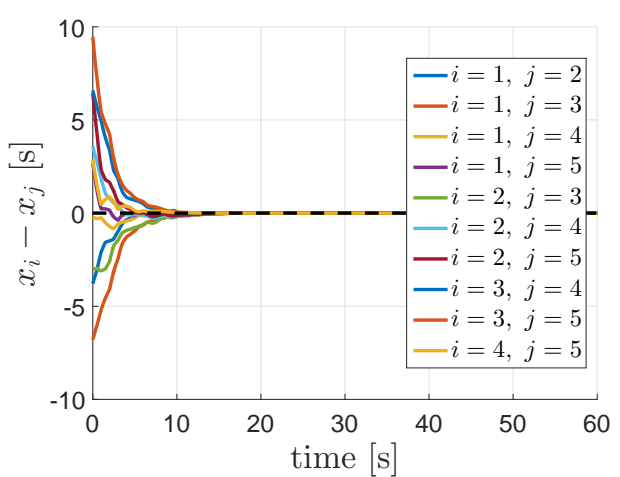

(a) Inter-vehicle coordination error, stringent coordination.

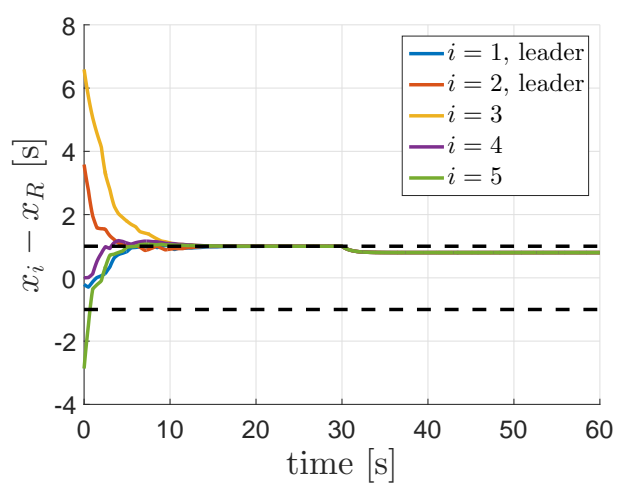

(c) Reference-vehicle coordination error, stringent coordination.

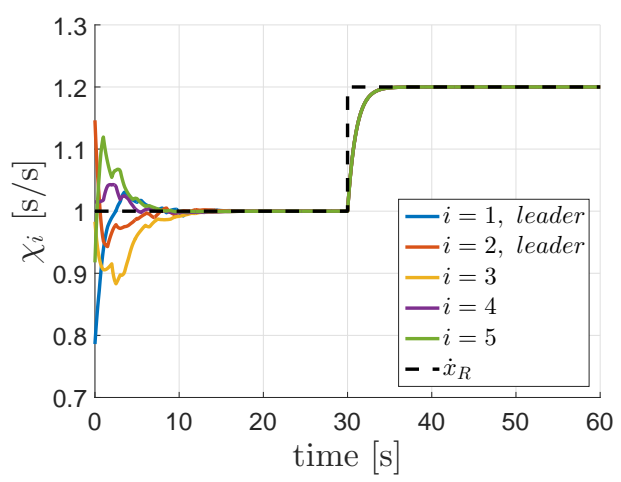

(e) Integral states, stringent coordination.

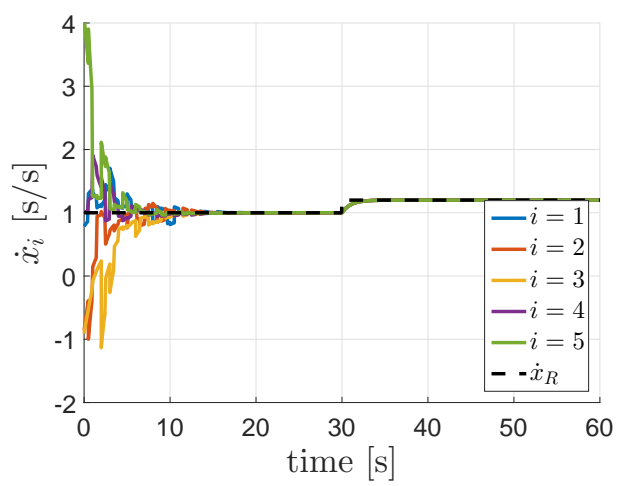

(g) Coordination-state rates, stringent coordination.

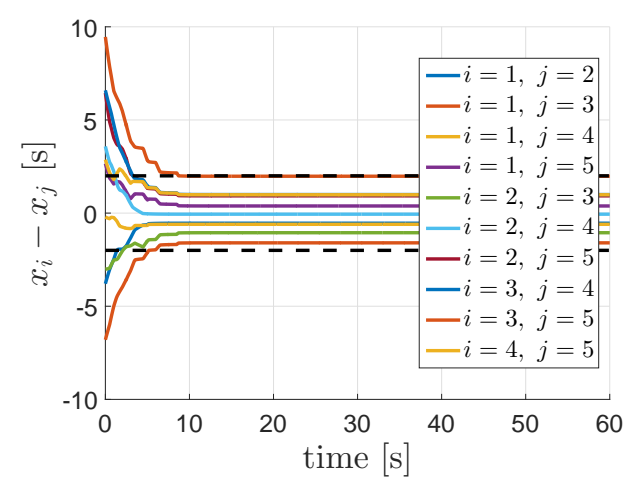

(b) Inter-vehicle coordination error, weak coordination.

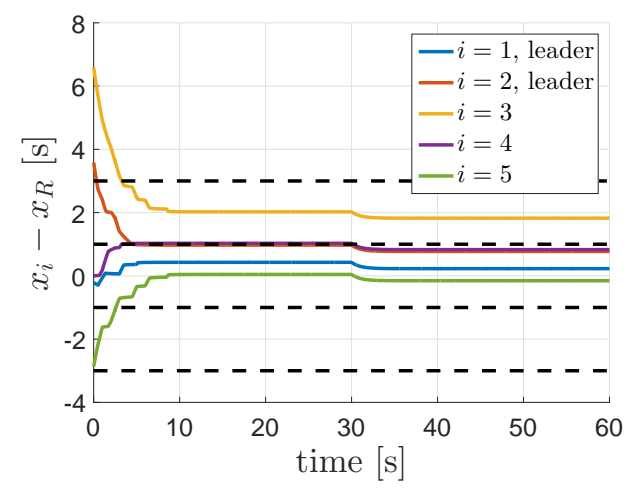

(d) Reference-vehicle coordination error, weak coordination.

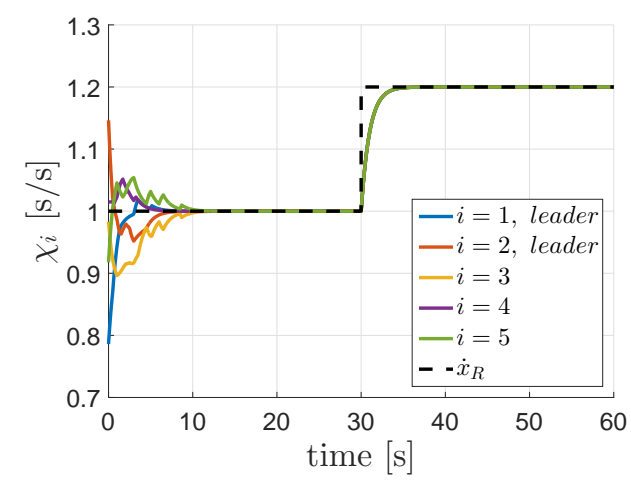

(f) Integral states, weak coordination.

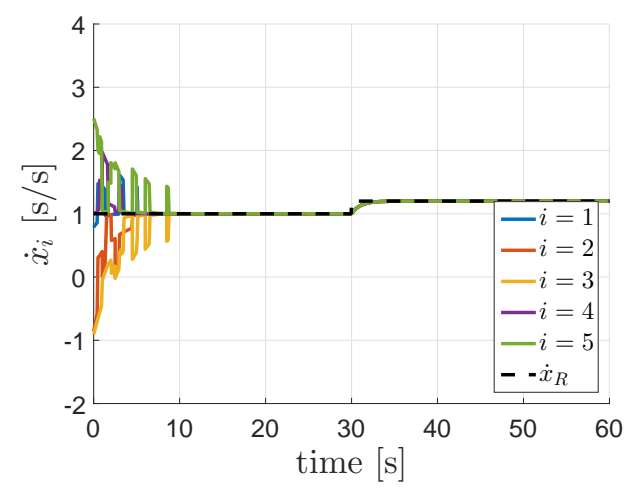

(h) Coordination-state rates, weak coordination

Figure 11: Loose temporal constraints. 